of BV in the genital specimens was assessed using Gram stain smear and Nugent scoring while that of Candida was assessed using microscopy. In-house multiplex PCR was used to detect the following STI pathogens - Neisseria gonorrhoeae (NG), Chlamydia trachomatis (CT), Trichomonas vaginalis (CT) and Mycoplasma genitalium (MG). HIV positivity was determined using commercial rapid tests.Data was captured into a Microsoft Access 2010 database and exported into Stata 14 for analysis using descriptive statistics, comparing four groups Group 1- women with BV or Candida infection only, Group 2 - women with BV or Candida with an STI, Group 3 women with an STI infection only and Group 4 - women with no pathogens detected in the laboratory.

Results A total of 771 women - median age 27 years [interquartile range (IQR) 23-32 years], 140 (19.5\%) $\geq 35$ years and $326(45.4 \%)$ HIV positive- were enrolled. Of these: 253 (35.3\%) were in Group 1, 238 (33.2\%) in Group 2, 99 $(13.8 \%)$ in Group 3, and $127(17.7 \%)$ in Group 4. The proportions of women who were 35 years or older were $20.2 \%$, $13.9 \%, 19.2 \%$ and $29.1 \%$ in Groups $1,2,3$ and 4 respectively $(\mathrm{p}=0.006)$ while the HIV positivity was $43.1 \%, 55.9 \%$, $43.4 \%$ and $32.3 \%$ in the groups $(\mathrm{p}<0.001)$. Condom use at last sexual encounter was $24.6 \%, 16.6 \%, 19.4 \%$ and $16.8 \%$ in Groups 1, 2, 3 and 4 respectively. The groups were similar in other respects although women in Group 2 had significantly higher rates of CT compared to Group 3.

Conclusion There were smaller but significant proportions of women 35 years or older had STIs with or without BV or Candida. The use of this age-specific threshold to guide syndromic treatment should be reconsidered, and HIV/STI integration promoted for women with VDS.

Support: The Centre for HIV and STIs at National Institute of Communicable Diseases in South Africa is funded by the South Africa's National Department of Health. No pharmaceutical grants were received in the development of this study.

\section{P3.219 EVOLUTION OF NEISSERIA GONORRHOEAE RESISTANCE TO ANTIMICROBIALS IN A HISTORICAL SERIES OF ISOLATES FROM SÃO PAULO/BRAZIL}

Thais Mattos Dos Santos'; Lisleia Golfetto'; Marcos André Schorner'; Hanalydia de Melo Machado'; Jessica Motta Martins'; Felipe De Rocco'; Ursula Oliveira Machado Souza'; Roberto José Carvalho Silva'; Maria Luiza Bazzo ${ }^{1}{ }^{1}$ Federal University of Santa Catarina, Florianópolis - SC, Brazil; ${ }^{2}$ STD/AIDS Reference and Training Centre of São Paulo, São Paulo $-S P$, Brazil

\subsection{6/sextrans-2017-053264.454}

Introduction: Neisseria gonorrhoeae (NG) was originally highly susceptible to antimicrobials. The ongoing selective pressure allied to the bacterium's ability to develop and retain resistance factors has been indicating the possibility of NG to evolve into untreatable forms. Therefore, constant monitoring of antimicrobial resistance and updates of treatment guidelines are mandatory. The aim of this study was to evaluate the evolution of NG antimicrobial resistance in a historical series of isolates from São Paulo/Brazil.

Methods A total of $173 \mathrm{NG}$ isolates obtained between 2003 and 2016 from the STD/AIDS Reference and Training Centre of São Paulo were analysed. The bacterial species identification was confirmed by MALDI-TOF and the minimum inhibitory concentration (MIC) for penicillin (PEN), tetracycline (TET), ciprofloxacin (CIP), azithromycin (AZT), ceftriaxone (CRO) and cefixime (CFX) was determined by agar dilution.

Results The isolates were divided into two periods, from 2003 to 2010 and 2011 to 2016 . The susceptibility profile for PEN did not differ between the two periods, $77.8 \%$ of the isolates had intermediate (I) and 19\% resistant (R) from 2003 to 2010, while $74.5 \%$ I and $25.5 \%$ R from 2011 to 2016. The susceptibility profile for TET showed a different behaviour with more intermediates in the second period $(59.6 \%$ I and $31.9 \%$ R) compared to the first period $(29.4 \%$ I and $60.3 \%$ $\mathrm{R})$. For AZT, there was an increase in the number of resistant isolates over the years: $0.8 \% \mathrm{I}$ and $1.6 \% \mathrm{R}$ (2003 to 2010), and $10.6 \%$ I and $12.8 \% \mathrm{R}$ (2011 to 2016). CIP was the drug that presented the major difference between the periods with $86.5 \%$ of isolates being sensitive, $1.6 \% \mathrm{I}$ and $11.9 \% \mathrm{R}$ in the first period and $46.8 \% \mathrm{R}$ in the second period. The isolates were $100 \%$ sensitive to cefixime and ceftriaxone.

Conclusion The study showed an increase in resistance to azithromycin and especially to ciprofloxacin. Ceftriaxone and cefixime isolates, both rarely used in clinic in Brazil, did not present resistance.

\section{P3.220 HUMAN PAPILLOMAVIRUS INFECTION IN ORAL AND ANOGENITAL SITES: PREVALENCE AND RATES OF CONCORDANCE}

Thaissa Cordeiro, Silvia Maria Baeta Cavalcanti. Universidade Federal Fluminense, Niteroi RJ, Brazil

\subsection{6/sextrans-2017-053264.455}

Introduction HPV infection causes cancer at several anatomical sites. However, the natural history of the infection in non-cervical sites have been understudied, especially at the oral epithelium.

Methods In our study, we investigated 351 samples from three different sites of 117 patients, searching for HPV By generic and specific PCR and Microarray, and related risk factors.

Results HPV DNA prevalence was $89.5 \%$ (105/117) in the genital lesions, $53.8 \%(63 / 117)$ in oral samples and 59\% $(69 /$ 117) in anal samples. Regarding the risk factors associated with HPV in the genital lesions, we found statistically significant rates for oral $(\mathrm{p}=0.039)$ and anal sex practices $(p=0.0000012)$. For oral samples, we observed a relevant correlation concerning oral contraceptive use $(p=0.039)$, tobacco smoking $(p=0.036)$ and alcohol use $(p=0.0075)$ while in anal samples, we found higher risk for HPV infection in patients relating non-exclusive sexual partners $(p=0.013)$. The presence of viral DNA in all the three sites was observed in $36.8 \%$ of the cases (43/117). Among them, 18\% (21/117) presented concordant HPV genotypes, diverging from the literature, corroborating that there is still much to learn about HPV natural history, since different biological behaviours are expected within different populations.

Conclusion In our study we also evaluated if the detection of oral HPV would suggest an infection in the anogenital tract. Nevertheless, our results showed only $36.8 \%$ of correlation pointing out that it is not suitable as a an auxiliary biomarker for HPV anogenital infections. 